\title{
Transverse correlation: An efficient transverse flow estimator - initial results
}

Holfort, Iben Kraglund; Henze, Lasse; Kortbek, Jacob; Jensen, Jørgen Arendt

Published in:

2008 IEEE Ultrasonics Symposium

Link to article, DOI:

10.1109/ULTSYM.2008.0395

Publication date:

2008

Document Version

Publisher's PDF, also known as Version of record

Link back to DTU Orbit

Citation (APA):

Holfort, I. K., Henze, L., Kortbek, J., \& Jensen, J. A. (2008). Transverse correlation: An efficient transverse flow estimator - initial results. In 2008 IEEE Ultrasonics Symposium (Vol. 1-4, pp. 1619-1622). IEEE. I E E E International Ultrasonics Symposium. Proceedings https://doi.org/10.1109/ULTSYM.2008.0395

\section{General rights}

Copyright and moral rights for the publications made accessible in the public portal are retained by the authors and/or other copyright owners and it is a condition of accessing publications that users recognise and abide by the legal requirements associated with these rights.

- Users may download and print one copy of any publication from the public portal for the purpose of private study or research.

- You may not further distribute the material or use it for any profit-making activity or commercial gain

- You may freely distribute the URL identifying the publication in the public portal 


\title{
Transverse Correlation: an Efficient Transverse Flow Estimator - Initial Results
}

\author{
Lasse Henze ${ }^{1}$, Iben Kraglund Holfort ${ }^{1}$, Jacob Kortbek ${ }^{12}$ and Jørgen Arendt Jensen ${ }^{1}$ \\ 1) Center for Fast Ultrasound Imaging, DTU Electrical Engineering, Bldg. 348, \\ Technical University of Denmark, DK-2800 Kgs. Lyngby, Denmark \\ 2) B-K Medical, Mileparken 34, 2730 Herlev, Denmark
}

\begin{abstract}
Color flow mapping has become an important clinical tool, for diagnosing a wide range of vascular diseases. Only the velocity component along the ultrasonic beam is estimated, so to find the actual blood velocity, the beam to flow angle has to be known. Because of the unpredictable nature of vascular hemodynamics, the flow angle cannot easily be found as the angle is temporally and spatially variant. Additionally the precision of traditional methods is severely lowered for high flow angles, and they breakdown for a purely transverse flow. To overcome these problems we propose a new method for estimating the transverse velocity component. The method measures the transverse velocity component by estimating the transit time of the blood between two parallel lines beamformed in receive. The method has been investigated using simulations performed with Field II. Using 15 emissions per estimate, a standard deviation of $1.64 \%$ and a bias of $1.13 \%$ are obtained for a beam to flow angle of 90 degrees. Using the same setup a standard deviation of $2.21 \%$ and a bias of $1.07 \%$ are obtained for a beam to flow angle of 75 degrees. Using 20 emissions a standard deviation of $3.4 \%$ and a bias of $2.06 \%$ are obtained at 45 degrees. The method performs stable down to a signal-to-noise ratio of $0 \mathrm{~dB}$, where a standard deviation of $5.5 \%$ and a bias of $1.2 \%$ is achieved.
\end{abstract}

\section{INTRODUCTION}

Color flow mapping has become an important clinical tool for diagnosing a wide range of vascular diseases. Traditional color flow mapping works by sending out a number of ultrasound pulses in a given direction and then estimating the time shift between the received signals, caused by the movement of the blood scatterers [1]. Only the velocity component along the ultrasonic beam is estimated, so to find the actual blood velocity, the beam to flow angle has to be known. Because of the unpredictable nature of vascular hemodynamics, the flow angle cannot easily be found. The precision of traditional methods is also lowered severely for high flow angles, and they break down for a purely transverse flow. A number of authors have proposed several solutions to this problem. Fox [2] suggested using two active apertures to get two independent beam to flow angles, making it possible to calculate the true velocity. For this to work, a large aperture has to be used, which in a clinical environment is unpractical. The precision of the method is also strongly dependent on the difference in beam to flow angle. This causes problems when imaging at large depths. Trahey [3] used a speckle tracking approach, where a $2 \mathrm{D}$ cross correlation between two consecutive images is used to estimate the velocity vector. This approach sets high demands on the beamformer of the imaging system, since a whole image has to be formed for every emission.
Jensen and Munk [4] used a dual Hanning weighting on the aperture to introduce a transverse oscillation. The method has shown promising in-vivo result [5], however a substantial number of emissions are needed to get an acceptable standard deviation. Anderson proposed a similar approach [6]. Jensen [7] suggested to find the velocity by beamforming lines along the direction of flow. To find the flow angle Kortbek and Jensen [8] suggested to beamform lines in a range of directions from 0 to 180 degrees and then search for the angle with the largest correlation over emissions. A low standard deviation is obtained, but the approach is very computational demanding. Hein [9] suggested using a time domain correlation technique to estimate the vector velocity. This approach is similar to the one proposed in this article, but sincea fixed geometric focus was used, the method was not able of imaging, and suffers from a poor SNR.

The method presented in this paper measures the transverse velocity component by estimating the transit time of the blood scatteres between two parallel lines beamformed in receive. Using the same data the axial velocity is also obtained, thus yielding the vector velocity. This approach enables imaging with a low number of computations making the method suitable for real time implementation.

\section{THEORY}

In the following section, it is assumed that the flow is moving from left to right and is spatially and temporally invariant. These assumptions are of course invalid for the flow encountered in the human vascular system, but as it will be obvious later in the paper, they are only introduced to ease the theoretical explanation.

The setup of the method can be seen in Fig. 1. The transducer is placed on top of the vessel, and a weakly focused beam is emitted by focusing below the estimation area. A total of $N$ pulses are used to create an estimate, where $N / 2$ are used to search for the transit time, and $N / 2$ are used to perform averaging. To estimate the velocity at a position given by $\vec{r}$, three lines with the length $l$ are beamformed. The center line $L_{Z}$ is used for estimating the axial velocity component. The center of $L_{Z}$ is positioned at $\vec{r}$. The two lines to the left and right from the center, $L_{A}$ and $L_{B}$, are used for estimating the transverse velocity. They are each beamformed a distance $d_{x}$ in the $x$ direction away from 


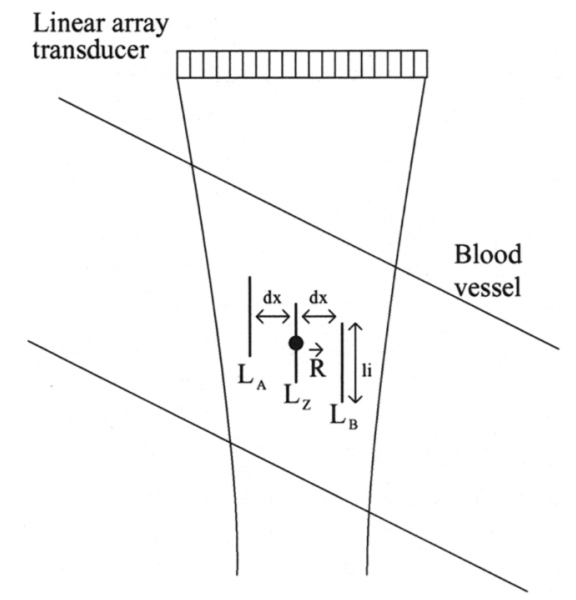

Fig. 1. Setup of the transverse velocity estimator. A linear array sends out a weakly focused beam in transmit. Three lines are beamformed in receive. The center line $L_{z}$ is used for estimating the axial velocity, the two lines to the side, $L_{A}$ and $L_{B}$ are used for estimating the transverse velocity. $L_{A}$ and $L_{B}$ are moved $d_{x}$ away from the center. All three lines have a length of $l$.

the center, as shown in the figure. The method estimates the axial velocity by using a cross-correlation technique [10].

Since the scatterers move from left to right with a spatially and temporally invariant velocity, the scatterers originally positioned in $L_{A}$ will eventually move into $L_{B} . L_{A}$ is therefore related to $L_{B}$ by a shift in time and axial position. To find the shift in time, a transit correlation function is created by calculating the normalized correlation coefficient between $L_{A}$ taken from emission $n$ with $L_{B}$ taken from emission $n$ to $n+N / 2-1$. The normalized correlation coefficient is given by

$$
C(a, b)=\max \left\{\frac{R_{a b}(\tau)-\mu_{a} \mu_{b}}{\sqrt{\left[R_{a a}(0)-\mu_{a}^{2}\right]\left[R_{b b}(0)-\mu_{b}^{2}\right]}}\right\}
$$

where $R_{a b}$ is the cross-corelation between signal $a$ and $b$, $\mu_{a}$ is the mean of $a$ and $\mu_{b}$ is mean of $b$. When the time span between $L_{A}$ and $L_{B}$ corresponds to the transit time of the blood between the two lines, the scatteres originally positioned in $L_{A}$ will now be positioned in $L_{B}$ resulting in a high correlation. The transit time can therefore be found by finding the peak of the transit correlation function. Since the distance between the lines is known, the transverse velocity component is given by

$$
V_{x \vec{r}}=\frac{2 d_{x}}{T_{t}}
$$

where $T_{t}$ is the estimated transit time. To further increase the performance of the method, a compensation for the axial velocity is applied by moving the two lines up and down according to the estimated axial velocity.

Fig. 2 shows a typical set of beamformed lines used for estimating the transverse velocity. The dotted line is the left line $L_{A}$, and the solid line is the right line $L_{B}$. The beam to flow angle is 75 degrees, the transverse velocity is $0.6 \mathrm{~m} / \mathrm{s}$, $L_{A}$ and $L_{B}$ are moved $0.2 \mathrm{~mm}$ away from the center, and a $f_{\text {prf }}$ of $3 \mathrm{kHz}$ is used. The lines are created by beamforming simulated data. The simulation is described in detail in section III-A. The $6 L_{A}$ lines are taken from emission 1, and the $6 L_{B}$ lines are taken from emission 1 to 6 . The axial shift caused by the axial velocity compensation can be observed on $L_{A}$ as an upward movement over emissions. It can be visually seen that the scatterers use 2 emissions to travel the distance from $L_{A}$ to $L_{B}$, since $L_{A}$ and $L_{B}$ are most similar when $L_{A}$ is taken from emission 1 , and $L_{B}$ is taken from emission 3 . Using (2) this corresponds to a transverse velocity of $0.6 \mathrm{~m} / \mathrm{s}$. This estimate matches the velocity used in the simulation.

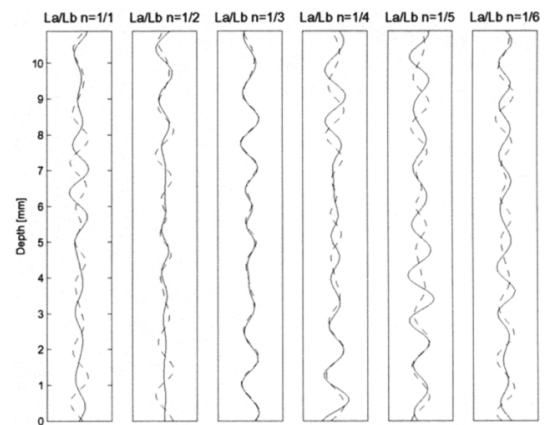

Fig. 2. Shows a set of lines used by the transverse velocity estimator. The dotted line is the left line $L_{A}$, and the solid line is the right line $L_{B}$ as seen in Fig. 1. The $L_{A}$ lines are taken from emission 1, and the $L_{B}$ lines are taken from emission 1 to 6 . The transit time for the scatterres are found as the instance when the two lines are most similar.

\section{METHODS}

\section{A. Simulations}

To evaluate the method, a series of simulations have been carried out under different situations. All the simulations have been made using the Field II program [11] [12]. The flow phantom has a laminar, parabolic velocity distribution given by

$$
v(r)=v_{0}\left(1-\left(\frac{r}{R}\right)^{2}\right),
$$

where $v_{0}$ is the peak velocity, $r$ is the radial position in the vessel, and $R$ is the radius of the vessel.

The flow phantom is created by placing point scatterers randomly over the region of interest. All the point scatterers are assigned a Gaussian distributed amplitude. The point scatters laying outside the vessel are given a amplitude that is $40 \mathrm{~dB}$ higher than the amplitude of the scatterers inside the vessel to mimic the situation encountered in a real scanning situation. The scatterers inside the vessel are moved between each pulse emission according to the spatial velocity field. The propagation of the scatters is given by 


$$
\vec{r}\left(t+T_{p r f}\right)=\vec{r}(t)+\vec{v}(\vec{r}(t)) T_{p r f}
$$

where $\vec{r}(t)$ is a vector defining the position of the scatterer at time $t, T_{p r f}$ is the time between pulse emissions, and $\vec{v}(\vec{r})$ is the velocity vector at position $\vec{r}$. I.

The basic parameters for the simulation are given in Table

TABLE I

STANDARD PARAMETERS For PARABOLIC FLOW SIMULATION AND VELOCITY ESTIMATION

\begin{tabular}{lcc}
\hline Transducer center frequency & $f_{0}$ & $7 \mathrm{MHz}$ \\
Speed of sound & $c$ & $1540 \mathrm{~m} / \mathrm{s}$ \\
Wavelength & $\lambda$ & $0.22 \mathrm{~mm}$ \\
Pitch of transducer elements & $w$ & $0.208 \mathrm{~mm}$ \\
Height of transducer elements & $h_{e}$ & $4.5 \mathrm{~mm}$ \\
Kerf & $\mathrm{ke}$ & $0.035 \mathrm{~mm}$ \\
Number of active elements(receive/transmit) & $N_{e}$ & $128 / 128$ \\
(Elevation focus) & $R_{e}$ & $25 \mathrm{~mm}$ \\
\hline RF lines for estimation & $N$ & 15 \\
Sampling frequency & $f_{s}$ & $40 \mathrm{MHz}$ \\
Pulse repetition frequency & $f_{p r f}$ & $3000 \mathrm{~Hz}$ \\
Number of oscillations & $N_{o}$ & 1 \\
Length of La and Lb & $l$ & $0.55 \mathrm{~mm}$ \\
Distance between La and Lb & $2 d_{x}$ & $0.4 \mathrm{~mm}$ \\
Correlation interval & $C_{i}$ & $\pm \frac{\lambda}{4}$ \\
Sampling interval for beamforming & $d_{b f}$ & $\lambda / 20$ \\
\hline Radius of vessel & $R$ & $8 \mathrm{~mm}$ \\
Distance to vessel center & $Z_{v e s}$ & $28 \mathrm{~mm}$ \\
Peak velocity & $v_{0}$ & $0.6 \mathrm{~m} / \mathrm{s}$ \\
Beam to flow angle & $B_{a}$ & $75 \mathrm{degrees}$ \\
\end{tabular}

\section{B. Results statistics}

To evaluate the performance of the method, the standard deviation and bias are calculated for all simulations. The bias is given by

$$
B_{\text {est }}\left(z_{k}\right)=v_{\mu}\left(z_{k}\right)-v_{\text {true }}\left(z_{k}\right),
$$

where $z_{k}$ is a discrete depth, $v_{\mu}$ is the mean of the estimates for the discrete depth, and $v_{\text {true }}$ is the true velocity. The standard deviation is given by

$$
\sigma_{e s t}(z k)=\sqrt{\frac{1}{N_{e s t}-1} \sum_{i=1}^{i=N_{e s t}}\left(v_{i}\left(z_{k}\right)-v_{\mu}\left(z_{k}\right)\right)^{2}},
$$

where $N_{\text {est }}$ is the number of estimates used for calculating the standard deviation, and $v_{i}$ is an estimate. To get out a single number representing the performance of a simulation, the mean and standard deviation is averaged over a range \pm $4 \mathrm{~mm}$ from the vessel center, and normalized with respect to the peak velocity $v_{0}$, as

$$
\begin{aligned}
& B_{r e l}=\frac{1}{v_{0} \cdot N_{z k}} \sum_{z_{k}=1}^{N_{z k}} B_{e s t}\left(z_{k}\right) \\
& \sigma_{r e l}=\frac{1}{v_{0} \cdot N_{z k}} \sum_{z_{k}=1}^{N_{z k}} \sigma_{e s t}\left(z_{k}\right)
\end{aligned}
$$

where $N_{z k}$ is the number of estimates in the interval. The outer most parts of the vessels are not included in the calculations of the statistical parameters to avoid wall effects.

Because the method relies on correlation, two types of errors are present [13]. The first, called a false peak error, is when a high sidelobe is identified by the estimator. This type of error is large, but can be removed by spatial or temporal median filtering [14]. The second type of error, called a jitter error, is a slight shift of the peak of the correlation function due to contamination of noise, finite sampling, etc. Since a few false peak errors will have a dominating effect on the calculation of the statistical parameters and they relative easily can be removed, a rejection scheme is used. Only estimates laying within $30 \%$ of the true velocity are included in the calculations of the statistical parameters. The percentage of rejected estimates will also be presented.

\section{RESULTS}

The top of Fig. 3 shows a plot of 25 transverse velocity profiles for a purely transverse flow. 15 emissions are used to create every profile, with a transmit focus in $56 \mathrm{~mm}$ and a correlation line length $l$ of $1.1 \mathrm{~mm}$. The bottom part of Fig. 3 shows the mean and standard deviation for the profile. The mean normalized standard deviation over the profile is $1.64 \%$, and the mean normalized bias is $1.13 \%$. No estimates have been left out due to the rejection scheme. The results show that the estimator works well for spatially variant velocity fields. The standard deviation is seen to increase at the sides of the simulated vessel. This is due to the steeper gradient of the velocity field, and the fact that the scatterers move to slowly to travel the distance between $L_{A}$ and $L_{B}$.
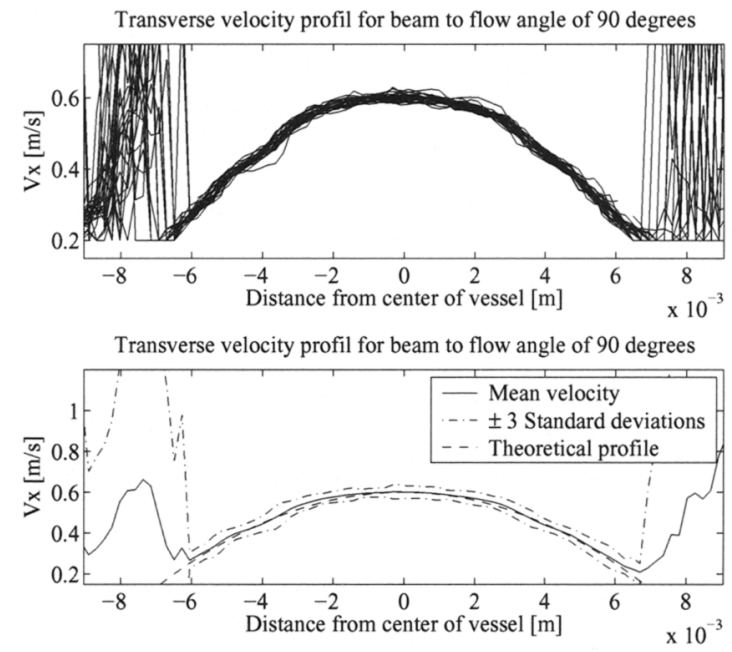

Fig. 3. The top of the figure shows 25 velocity profiles for a beam to flow angle of 90 degrees. The bottom of the figure shows, the mean profile (full) together with \pm 3 standard deviation (dashdot) and the the theoretical profile(dashed).

The top part of Fig. 4 shows a plot of 24 velocity profiles for a beam to flow angle of 45 degrees. 20 emissions are 
used to create each profile, with focus in $56 \mathrm{~mm}$ and a correlation line length $l$ of $0.55 \mathrm{~mm}$. The bottom part of Fig. 4 shows the mean and standard deviation for the profile. The normalized mean bias over the velocity profile is $2.06 \%$, and the mean normalized standard deviation is $3.40 \%, 4.6 \%$ of the estimates have been left out due to the rejection scheme. The result shows that the method is capable of producing satisfactory results for a low beam to flow angle. Fig. 5 shows
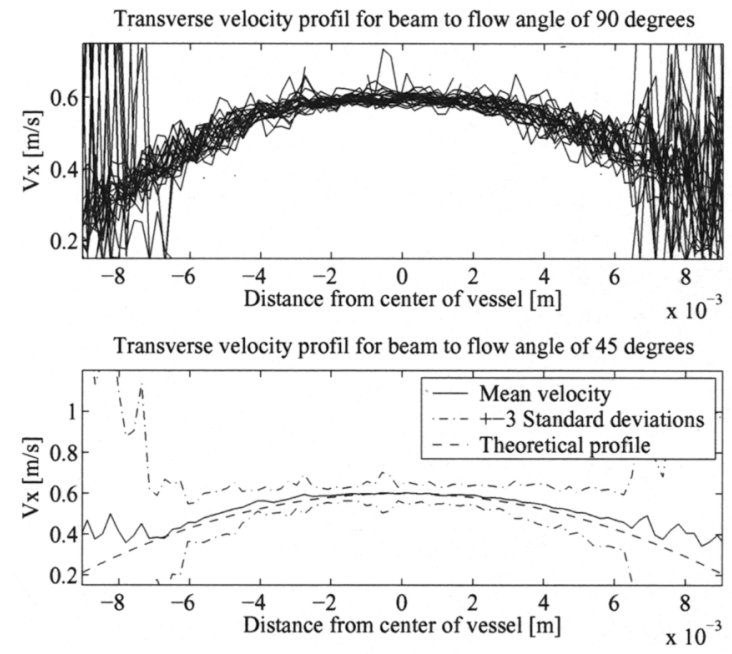

Fig. 4. The top of the figure shows 24 velocity profiles for a beam to flow angle of 45 degrees. The bottom of the figure shows, the mean profile (full) together with \pm 3 standard deviation (dashdot) and the the theoretical profile(dashed).

the performance of the method for different SNR values. The SNR is defined by,

$$
S N R_{d B}=10 \log _{10} \frac{E\left\{g_{b}^{2}(t)\right\}}{E\left\{n^{2}(t)\right\}},
$$

where $g_{b}(t)$ is the beamformed signal taken from the middle of the vessel, and $n(t)$ is the noise. The SNR is varied from -10 to $30 \mathrm{~dB}$. The standard deviation falls exponentially until it reaches a plateau of around $3 \%$ for a SNR of $10 \mathrm{~dB}$ or greater. More than $28 \%$ of the estimates are rejected, when the SNR gets below 4 .

\section{CONCLUSION}

A new transverse velocity estimator has been proposed. Realistic flow simulations have been carried out to test its performance. The estimator is robust and has been able to measure the transverse velocity component with a low standard deviation without any considerable bias.

\section{ACKNOWLEDGMENT}

This work was supported by the Faculty of Health Science, University of Copenhagen, by grant 274-05-0327 from the Danish Research Agency, the Radio-parts foundation and by B-K Medical Aps, Denmark.
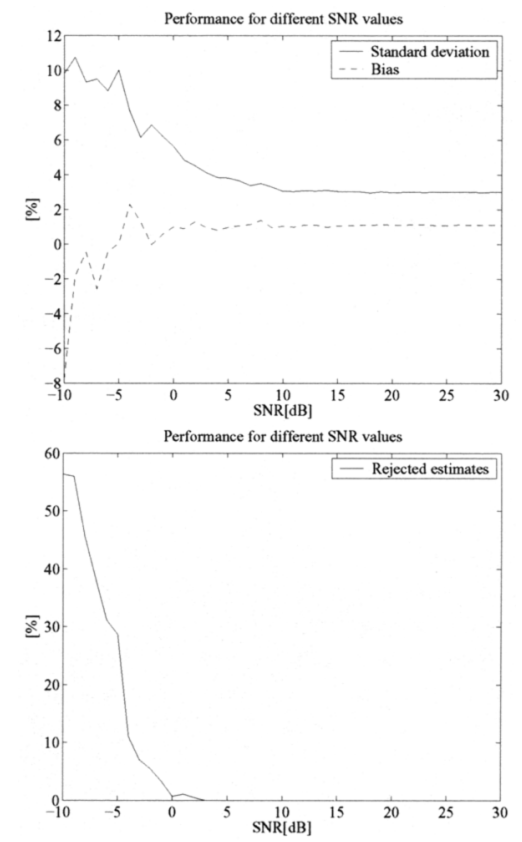

Fig. 5. Variations in SNR

\section{REFERENCES}

[1] J. A. Jensen, Estimation of Blood Velocities Using Ultrasound: A Signal Processing Approach, Cambridge University Press, New York, 1996.

[2] M. D. Fox, "Multiple crossed-beam ultrasound Doppler velocimetry," IEEE Trans. Son. Ultrason., vol. SU-25, pp. 281-286, 1978.

[3] G. E. Trahey, J. W. Allison, and O. T. von Ramm, "Angle independent ultrasonic detection of blood flow," IEEE Trans. Biomed. Eng., vol. BME-34, pp. 965-967, 1987.

[4] J. A. Jensen and P. Munk, "A new method for estimation of velocity vectors," IEEE Trans. Ultrason., Ferroelec., Freq. Contr., vol. 45, pp. $837-851,1998$.

[5] J. Udesen, F. Gran, and J. A. Jensen, "A frequency splitting method for CFM imaging," in Proc. IEEE Ultrason. Symp., 2006, p. Accepted for publication.

[6] M. E. Anderson, "Multi-dimensional velocity estimation with ultrasound using spatial quadrature," IEEE Trans. Ultrason., Ferroelec., Freq. Contr., vol. 45, pp. 852-861, 1998.

[7] J. A. Jensen, "Directional velocity estimation using focusing along the flow direction: I: Theory and simulation," IEEE Trans. Ultrason., Ferroelec., Freq. Contr., pp. 857-872, 2003.

[8] J. Kortbek and J. A. Jensen, "Estimation of velocity vector angles using the directional cross-correlation method," IEEE Trans. Ultrason., Ferroelec., Freq. Contr., vol. 53, pp. 2036-2049, 2006.

[9] Ilmar Hein, "3-d flow velocity vector estimation with a triple-beam lens transducer-experimental results," IEEE Trans. Ultrason., Ferroelec., Freq. Contr., vol. 44, pp. 85-95, 1997.

[10] O. Bonnefous and P. Pesqué, "Time domain formulation of pulseDoppler ultrasound and blood velocity estimation by cross correlation," Ultrason. Imaging, vol. 8, pp. 73-85, 1986

[11] J. A. Jensen, "Field: A program for simulating ultrasound systems," Med. Biol. Eng. Comp., vol. 10th Nordic-Baltic Conference on Biomedical Imaging, Vol. 4, Supplement 1, Part 1, pp. 351-353, 1996b.

[12] J. A. Jensen and N. B. Svendsen, "Calculation of pressure fields from arbitrarily shaped, apodized, and excited ultrasound transducers," IEEE Trans. Ultrason., Ferroelec., Freq. Contr., vol. 39, pp. 262-267, 1992.

[13] W. F. Walker and G. E. Trahey, "A fundamental limit on the performance of correlation based phase correction and flow estimation techniques," IEEE Trans. Ultrason., Ferroelec., Freq. Contr., vol. 41, pp. 644-654, 1994.

[14] J. A. Jensen, "Range/velocity limitations for time-domain blood velocity estimation," Ultrasound Med. Biol., vol. 19, pp. 741-749, 1993c. 